\title{
Analysis of adiscrete- time single server retrial queue with general retrial times, multiple vacations and statedependent arrivals
}

\author{
N. Kalpana ${ }^{1 *}$, R. Arumuganathan ${ }^{2}$ \\ 1 *Corresponding Author: Department of Science and Humanities, Coimbatore Institute of Engineering and \\ Technology, Coimbatore-641109, India. \\ ${ }^{2}$ Department of Mathematics, PSG College of Technology, Coimbatore - 641004, Tamil Nadu, India.
}

\begin{abstract}
This paper analyses a discrete time Geo/G/1 retrial queuing system with general retrial times, multiple vacations and state dependent arrivals. Here the arrival rates are different when the system is idle and busy or on vacation. If the server is busy or on vacation at the arrival epoch, the customers joins the orbit to repeat the request later. On the other hand, if the server is idle, then the arriving customer begins its service immediately. The customers in the orbit try for service when the server is idle. At a service completion epoch, if the number of customers in the orbit is zero, the server goes for vacation repeatedly until at least one customer is found in the orbit. At a vacation completion epoch, if there is at least one customer found in the orbit, then the server remains idle in the system to render service for customers either from the main pool or from the retrial group. The primary arrival rate is $p_{1}$ when the server is idle and the primary arrival rate is $p_{2}$ when the server is busy or on vacation $\left(p_{1}>p_{2}\right)$. The Steady state probabilities of the considered queueing system are analysed. Performance measures through the generating functions are obtained. Stochastic decompositionof thesystem size is obtained without vacation and the additional system size due to vacation. Numerical results with graphs are provided to study the performance measures of the system.
\end{abstract}

Keywords: Discrete time retrial queues, general retrial times, state dependent arrivals, multiple vacation, Markov chain.

\section{Introduction}

There is a great potential for using the discrete time queues in the performance analyses of computer and communication networks. Discrete time queueing system is an efficient tool to model the advancement in the telecommunication systems because of its wide range of application. In the past few years, queueing models in discrete time have been analysed by many researchers, details of which may be found in books of Takagi [16] and Hunter [10]. Discrete time retrial queues have been widely used to model many practical problems such as digital communication systems and telecommunication networks like broad band integrated services Digital Network (B-ISDN), which includes Asynchronous transfer mode (ATM) based system, and circuit switched time division multiple access (TDMA). For the detailed overview the reader may refer bibliography on retrial queues by Artalejo in [1], [2] and [3] and Falin and Templeton in [8] and [9]. Queueing systems with repeated attempts are characterized by the fact that a customer finding the server busy upon arrival must leave the service area and join a retrial group (orbit) in order to retry its request after some random time. For detailed overview refer Yang and Templeton [18].

In the past, the study of the retrial queues has been focussed on the continuous time systems. But Yang and $\mathrm{Li}[15]$ were the first to discuss the discrete-time Geo/G/1 retrial queues.The model of Yang and Li was generalized by Atencia and Moreno [7] to the discrete-time Geo/G/1retrial queue with general retrial times. Refer to [4], [5] and [6] for related literature on discrete-time retrial queueing systems.

Queueing systems with server vacation are very useful in systems where the server can utilise the idle time for other purposes. It is extensively used in computer networks and production management. For detailed overview of vacation queueing models refer [17]. Retrial queue with a finite number of input sources and server vacations was studied by $\mathrm{Li}$ and Yang [14] and they derived a recursive formula for the steady state probability and performance measures of the systemby using supplementary variable technique. Ke and Chang [13] analysed a modified vacation policy for M/G/1 retrial queue with balking and feedback andKe and Chu [12] discussed a modified vacation model for $\mathrm{M}^{\mathrm{x}} / \mathrm{G} / 1$ system.Zhang and Tian [19] study the discretetime Geo/G/1 queue with multiple adaptive vacations, whichincludes multiple-vacation and single-vacation models as its special cases.

Most of the retrial queuing papers with vacations have analysedsystems with single type of arrival. However, the analysis of discrete- time Geo/G/1 retrial queues with different arrival rates is quite interesting.This paper analysis a discrete- time single server retrial queue with general retrial times, multiple 
vacations and state dependent arrivals. As referred in [11], this queueing model is observed in packet switching network. The router is an inter-connecting device which attaches two or more networks, which receives packets and forwards it to the next hop. If the router is idle, a packet immediately receives service; otherwise the packets queues up in the buffer. When two types of packets (regular or urgent) arrive at the router, if the router is idle then more number of packets will be processed. If the system is busy or on vacation then the arrival is less. When the router is idle, to ensure effective functioning of the router, some maintenance activities such as routing information backup and virus scan will be performed.

The remaining sectionsof this paper is organized as follows. In Section II, the mathematical description of the model is developed. In section III, Markov chain and generating function analysis is presented. The distribution of the number of customers in the orbit and in system is derived. The performance measures of the system are obtained in section IV. In section V, stochastic decomposition result is obtained and in section VI, some numerical results and graphs are presented to illustrate the effect of parameters on several performance characteristics.

\section{Model Description}

Consider a single server discrete-time retrial queue. The time axis is segmented into slots marked by 0 , $1,2, \ldots, m, \ldots$ of equal length. All the queueing activities occur at the slot boundaries. In this queueing model, we consider an early arrival system (EAS) policy where we assume that the departures and the end of the vacations occur in the interval $\left(m^{-}, \mathrm{m}\right)$, and the arrivals, the retrials, and the beginningof thevacation occur in the interval $\left(m, \mathrm{~m}^{+}\right)$in sequence.

Customers arrive according to a geometric arrival process at the rate ' $p_{1}$ ' when the server is idle and with rate ' $p_{2}$ ' when the server is busy or on vacation $\left(p_{1}>p_{2}\right)$. There is no waiting space in the system. If an arriving customer finds that the server is idle, it begins the service immediately and leaves the system forever after service completion. Otherwise, if an arriving customer finds that the server is busy or on vacation, he joins the orbit. Successive inter-retrial times of any customer follow an arbitrary distribution $\left\{a_{i}\right\}$ with generating function $\mathrm{A}(x)=\sum_{i=0}^{\infty} a_{i} x^{i}$.

At a service completion epoch, if the number of customers in the orbit is zero, the server leaves for vacation repeatedly until at least one customer is found in the orbit. At a vacation completion epoch, if there is at least one customer found in the orbit, then the server remains idle in the system to render service for customers either from the main pool or from the retrial group.

The service times are independent and identically distributed with probability distribution $\left\{s_{i}\right\}$ and probability generating function $S(x)=\sum_{i=1}^{\infty} s_{i} x^{i}$ with the nth factorial moment $S_{\mathrm{n}}$. The vacation time is assumed to follow a general probability distribution $\left\{v_{i}\right\}$ with probability generating function $V(x)=\sum_{i=1}^{\infty} v_{i} x^{i}$ with the nth factorial moment $V_{n}$.

\section{The Markov Chain}

At time ${ }^{+}$, let $C_{m}$ denote the state of the server and $N_{m}$ denote the number of customers in the orbit, where $C_{m}=0,1$ or 2 according to whether the server is free, busy or on vacation. If $C_{m}=0$, let $\xi_{m}$ represents the remaining retrial time.If $C_{m}=1$, let $\xi_{\mathrm{m}}$ represents the remaining service time of the customer currently being served. If $C_{m}=2$, let $L_{m}=n$ represents that the server takes the nth vacation and let $\xi_{m}$ corresponds to the remaining vacation time.

$Y_{m}=\left\{C_{m}, L_{m}, \xi_{m}, N_{m}\right\}, \mathrm{m}=0,1,2, \ldots .$. is a Markov chain with the following state space $\{(0,0) ;(0, i, k): i \geq 1, k \geq 1 ;(1, i, k): i \geq 1, k \geq 0 ;(2, n, i, k): i \geq 1, k \geq 0, n \geq 1\}$

Define the stationary probabilities of the Markov chain $\left\{Y_{m}, m=0,1,2, \ldots.\right\}$ as follows.

$$
\pi_{0,0}=\lim _{m \rightarrow \infty} P\left\{C_{m}=0, N_{m}=0\right\}
$$

$\pi_{0, i, k}=\lim _{m \rightarrow \infty} P\left\{C_{m}=0, \xi_{m}=i, N_{m}=k\right\} ; i \geq 1, k \geq 1$

$\pi_{1, i, k}=\lim _{m \rightarrow \infty} P\left\{C_{m}=1, \xi_{m}=i, N_{m}=k\right\} ; i \geq 1, k \geq 0$

$\omega_{n, i, k}=\lim _{m \rightarrow \infty} P\left\{C_{m}=2, L_{m}=n, \xi_{m}=i, N_{m}=k\right\} ; i \geq 1, k \geq 0, n \geq 1$

Then, the Kolmogorov equations are obtained as follows.

$$
\begin{aligned}
& \pi_{0,0}=\bar{p}_{1} \pi_{0,0}+\bar{p}_{2} \sum_{n=1}^{\infty} \omega_{n, 1, k} \\
& \pi_{0, i, k}=\bar{p}_{1} \pi_{0, i+1, k}+\bar{p}_{2} a_{i} \pi_{1,1, k}+\bar{p}_{2} a_{i} \sum_{n=1}^{\infty} \omega_{n, 1, k} \quad i \geq 1, k \geq 1 \\
& \quad \pi_{1, i, k}=\delta_{0, k} p_{1} s_{i} \pi_{0,0}+\left(1-\delta_{0, k}\right) p_{1} s_{i} \sum_{j=1}^{\infty} \pi_{0, j, k}+\bar{p}_{1} s_{i} \pi_{0,1, k+1}+p_{2} s_{i} \pi_{1,1, k}+\bar{p}_{2} a_{0} s_{i} \pi_{1,1, k+1}+ \\
& \left(1-\delta_{0, k}\right) p_{2} \pi_{1, i+1, k-1}+\bar{p}_{2} \pi_{1, i+1, k}+\bar{p}_{2} a_{0} s_{i} \sum_{n=1}^{\infty} \omega_{n, 1, k+1}+p_{2} s_{i} \sum_{n=1}^{\infty} \omega_{n, 1, k} i \geq 1, k \geq 0(3) \\
& \omega_{1, i, k}=\bar{p}_{2} \omega_{1, i+1, k}+\left(1-\delta_{0, k}\right) p_{2} \omega_{1, i+1, k-1}+\delta_{0, k} \bar{p}_{2} v_{i} \pi_{1,1,0} \quad i \geq 1, k \geq 0(4)
\end{aligned}
$$


$\omega_{n, i, k}=\bar{p}_{2} \omega_{n, i+1, k}+\left(1-\delta_{0, k}\right) p_{2} \omega_{n, i+1, k-1}+\delta_{0, k} \bar{p}_{2} v_{i} \omega_{n-1,1,0} i \geq 1, k \geq 0, n \geq 2(5)$

where $\delta_{i, j}$ denotes the Kronecker delta defined as $\delta_{0, k}=\left\{\begin{array}{l}1, k=0 \\ 0, k \neq 0\end{array}\right.$, and the normalising condition is

$\pi_{0,0}+\sum_{i=1}^{\infty} \sum_{k=1}^{\infty} \pi_{0, i, k}+\sum_{i=1}^{\infty} \sum_{k=0}^{\infty} \pi_{1, i, k}+\sum_{n=1}^{\infty} \sum_{i=1}^{\infty} \sum_{k=0}^{\infty} \omega_{n, i, k}=1$

To solve Equations (1) - (5), we introduce the following generating functions:

$$
\varphi_{0}(x, z)=\sum_{i=1}^{\infty} \sum_{k=1}^{\infty} \pi_{0, i, k} x^{i} z^{k} \quad, \quad \varphi_{1}(x, z)=\sum_{i=1}^{\infty} \sum_{k=0}^{\infty} \pi_{1, i, k} x^{i} z^{k}
$$

$\varphi_{2}(x, z)=\sum_{n=1}^{\infty} \psi_{n}(x, z)$, where $\psi_{n}(x, z)=\sum_{i=1}^{\infty} \sum_{k=0}^{\infty} \omega_{n, i, k} x^{i} z^{k}, \mathrm{n} \geq 1$

and the auxiliary generating functions: $\varphi_{0, i}(z)=\sum_{k=0}^{\infty} \pi_{0, i, k} z^{k}, i \geq 1, \quad \varphi_{1, i}(z)=\sum_{k=0}^{\infty} \pi_{1, i, k} z^{k}, i \geq$ $1, \quad \psi_{n, i}(z)=\sum_{k=0}^{\infty} \omega_{n, i, k} z^{k}, i \geq 1, n \geq 1$

By multiplying Equations(2)-(5) by $\mathrm{z}^{\mathrm{k}}$ and summing over $\mathrm{k}$, the following equations are obtained:

$$
\begin{gathered}
\varphi_{0, i}(z)=\bar{p}_{1} \varphi_{0, i+1}(z)+\bar{p}_{2} a_{i} \varphi_{1,1}(z)-\bar{p}_{2} a_{i} \pi_{1,1,0}+\bar{p}_{2} a_{i} \sum_{n=1}^{\infty} \psi_{n, 1}(z)-\overline{p_{2}} a_{i} \sum_{n=1}^{\infty} \omega_{n, 1,0}, i \\
\geq 1 \quad(6) \varphi_{1, i}(z) \\
=p_{1} s_{i} \pi_{0,0}+p_{1} s_{i} \varphi_{0}(1, z)+\frac{\bar{p}_{1}}{z} s_{i} \varphi_{0,1}(z)+p_{2} s_{i} \varphi_{1,1}(z)+\frac{\bar{p}_{2}}{z} a_{0} s_{i}\left(\varphi_{1,1}(z)-\pi_{1,1,0}\right)+ \\
\left(\bar{p}_{2}+p_{2} z\right) \varphi_{1, i+1}(z)+\left(p_{2}+\frac{\bar{p}_{2} a_{0}}{z}\right) s_{i} \sum_{n=1}^{\infty} \psi_{n, 1}(z)-\frac{\bar{p}_{2}}{z} a_{0} s_{i} \sum_{n=1}^{\infty} \omega_{n, 1,0} \quad i \geq 1, k \geq 0(7) \\
\psi_{1, i}(z)=\left(\bar{p}_{2}+p_{2} z\right) \psi_{1, i+1}(z)+\bar{p}_{2} v_{i} \pi_{1,1,0} i \geq 1 \quad k \geq 0(8) \quad i \geq 1, k \geq 0, n \geq 2(9) \\
\psi_{n, i}(z)=\left(\bar{p}_{2}+p z\right) \psi_{1, i+1}(z)+\bar{p}_{2} v_{i} \omega_{n-1,1,0} \quad i \geq
\end{gathered}
$$

Now multiply the equations (8) and (9) by $x^{\mathrm{i}}$, and summing over ' $\mathrm{i}$ ' we get

$\frac{x-\Lambda(z)}{x} \psi_{1}(x, z)=-\Lambda(z) \psi_{1,1}(z)+\bar{p}_{2} V(x) \pi_{1,1,0}$

$\frac{x-\Lambda(z)}{x} \psi_{n}(x, z)=-\Lambda(z) \psi_{n, 1}(z)+\bar{p}_{2} V(x) \omega_{n-1,1,0}$

where $\Lambda(z)=\bar{p}_{2}+p_{2} z$

Setting $x=\Lambda(z)$ in equations (10) and (11),we get

$$
\psi_{1,1}(z)=\frac{\bar{p}_{2} V(\Lambda(z))}{\Lambda(z)} \pi_{1,1,0}
$$

$\psi_{n, 1}(z)=\frac{\overline{p_{2}} V(\Lambda(z))}{\Lambda(z)} \omega_{n-1,1,0} n \geq 2$

Substituting for $\psi_{1,1}(z)$ and $\psi_{n, 1}(z)$ in (10) and (11), we get

$\psi_{1}(x, z)=\frac{\overline{p_{2}} x[V(x)-V(\Lambda(z))]}{x-\Lambda(z)} \pi_{1,1,0}$

$\psi_{n}(x, z)=\frac{\overline{p_{2} x}[V(x)-V(\Lambda(z))]}{x-\Lambda(z)} \omega_{n-1,1,0} \quad n \geq 2$

Taking the derivative about $x$ to the equations (12) and (13) and letting $x=z=0$, we get 
$\omega_{1,1,0}=V\left(\bar{p}_{2}\right) \pi_{1,1,0}=M \pi_{1,1,0} \quad$ whereV $\left(\bar{p}_{2}\right)=M$

$\omega_{n, 1,0}=M \omega_{n-1,1,0}=M^{n} \pi_{1,1,0}, \quad n \geq 2(15)$

Substituting (15) into (1),we get

$\pi_{1,1,0}=\frac{p_{1} \pi_{0,0}}{\overline{p_{2} \sum_{n=1}^{\infty} M^{n}}}=\frac{p_{1} \pi_{0,0}}{\overline{p_{2} \vartheta_{2}}}$

Multiply Equations(6) and(7) by $x^{i}$,summing overi, we get

$\frac{x-\bar{p}_{1}}{x} \varphi_{0}(x, z)=\bar{p}_{2}\left(A(x)-a_{0}\right) \varphi_{1,1}(z)-\bar{p}_{1} \varphi_{0,1}(z)-$
$p_{1}\left(A(x)-a_{0}\right) \frac{\vartheta_{1}\left[\Lambda(z)-\bar{p}_{2} V(\Lambda(z))\right]}{\vartheta_{2} \Lambda(z)} \pi_{0,0} \quad(17)$
$\frac{x-\Lambda(z)}{x} \varphi_{1}(x, z)=$
$\left[\frac{p_{2} z+\bar{p} a_{0}(1-z)}{z} S(x)-\Lambda(z)\right] \varphi_{1,1}(z)+\frac{\bar{p}_{1}(1-z)}{z} S(x) \varphi_{0,1}(z)+$
$\frac{z\left[\Lambda(z)+p_{2} V(\Lambda(z))\right]-a_{0} \tau(z)}{z \Lambda(z) \vartheta_{2}} p_{1} S(x) \pi_{0,0}$

where $\vartheta_{1}=\left(1-M^{n}\right) /(1-M), \vartheta_{2}=\left(M-M^{n}\right) /(1-M), \tau(z)=\vartheta_{1}\left[\Lambda(z)-\bar{p}_{2} V(\Lambda(z))\right]$

$\varphi_{0}(1, z)$ and $\varphi_{1}(1, z)$ are obtained by substituting $x=1$ in equation (17) and (18).

Setting $x=\bar{p}$ in (17) and $x=\Lambda(z)$ in (18) and solving the equations, we get the generating functions as follows $\varphi_{0,1}(z)=$

$p_{1} z\left(A\left(\bar{p}_{1}\right)-a_{0}\right) \frac{\left[\left(p_{2} z-\bar{p}_{2} a_{0}\right)\left(\Lambda(z)-\bar{p}_{2} V(\Lambda(z))\right)+z\left[\Lambda(z)+p_{2} V(\Lambda(z))\right] S(\Lambda(z))-z \Lambda(z)\left(\Lambda(z)+p_{2} V(\Lambda(z))\right) \vartheta_{1}\right.}{\gamma(z) \vartheta_{2}} \frac{\pi_{0,0}}{\bar{p}_{1}}(20)$

$\varphi_{1,1}(z)=\frac{z\left[\Lambda(z)+p_{2} V(\Lambda(z))\right] \vartheta_{1}+\left(A\left(\bar{p}_{1}\right)(1-z)+a_{0} z\right) \tau(z)}{\gamma(z) \Lambda(z) \vartheta_{2}} p_{1} S(\Lambda(z)) \pi_{0,0}(21)$

where $\gamma(z)=\left[\left(\bar{p}_{2}\right) A\left(\bar{p}_{1}\right)(1-z)+z p_{2}\right] S(\Lambda(z))-z \Lambda(z)$

The following Lemmas are stated to obtain the probability generating function of the system size.

\section{Lemma 1:}

If $\rho<p_{2}+\bar{p}_{2} A\left(\bar{p}_{1}\right)$ then, $\left[\left(\bar{p}_{2}\right) A\left(\bar{p}_{1}\right)(1-z)+z p_{2}\right] S(\Lambda(z))-z \Lambda(z)>0$ where $\rho=p_{2} S_{1}$ and $0 \leq z<1$.

Proof: Let us define the following functions:

$f(z)=\left[\left(\bar{p}_{2}\right) A\left(\bar{p}_{1}\right)(1-z)+z p_{2}\right] S(\Lambda(z))$ and $g(z)=z \gamma(z)$ for $0 \leq z<1$.

We observe thatf ${ }^{\prime}(1)<\mathrm{g}^{\prime}(1)$, is equivalent to $\rho<p_{2}+\bar{p}_{2} A\left(\bar{p}_{1}\right)$. Also $\mathrm{f}(\mathrm{z})>g(\mathrm{z})$ in $0 \leq \mathrm{z}<1$, due to the fact that $\mathrm{f}(\mathrm{z})$ and $\mathrm{g}(\mathrm{z})$ are convex functions.

\section{Lemma 2:Stability Condition}

The following limits exist and are positive iff $\rho<p_{2}+\overline{p_{2}} A\left(\overline{p_{1}}\right)$

$$
\begin{gathered}
\lim _{\mathrm{z} \rightarrow 1} \frac{\left[\left(p_{2} z-\bar{p}_{2} a_{0}\right)\left(\Lambda(z)-\bar{p}_{2} V(\Lambda(z))\right)+z\left[\Lambda(z)+p_{2} V(\Lambda(z))\right] S(\Lambda(z))-z \Lambda(z)\left(\Lambda(z)-\bar{p}_{2} V(\Lambda(z))\right) \vartheta_{1}\right.}{\gamma(z)} \\
=\frac{\bar{p}_{2} V_{1} \vartheta_{1}+\left(1+p_{2}\right) \vartheta_{1} \rho-\bar{p}_{2} \vartheta_{1}}{p_{2}+\bar{p}_{2} A\left(\bar{p}_{1}\right)-\rho} \\
\lim _{\mathrm{z} \rightarrow 1} \frac{z\left[\Lambda(z)+p_{2} V(\Lambda(z))\right] \vartheta_{1}+\left(A\left(\bar{p}_{1}\right)(1-z)+a_{0} z\right) \tau(z)}{\gamma(z)}=\frac{A\left(\bar{p}_{1}\right)\left(1+p_{2}\right) \vartheta_{1}+p_{2}\left(V_{1}-1\right) \vartheta_{1}}{p_{2}+\bar{p}_{2} A\left(\bar{p}_{1}\right)-\rho}
\end{gathered}
$$

From the above Lemmas, it is observed that if $\rho<p_{2}+\bar{p}_{2} A\left(\bar{p}_{1}\right)$ is a necessary condition for the ergodicity of the Markov chain. Also $\varphi_{0,1}(\mathrm{z}), \varphi_{1,1}(\mathrm{z})$ are defined for $0 \leq z<1$ and can be extended by continuity in $\mathrm{z}=1$. 
The stationary distributionof the Markov Chain has the following generating functions:

$\varphi_{0}(x, z)=\frac{A(x)-A\left(\overline{p_{1}}\right)}{x-\overline{p_{1}}} \frac{\left[\left(p_{2} z-\overline{p_{2}} a_{0}\right)\left(\Lambda(z)-\overline{p_{2}} V(\Lambda(z))\right)+z\left[\Lambda(z)+p_{2} V(\Lambda(z))\right] S(\Lambda(z))-z \Lambda(z)\left(\Lambda(z)-\overline{p_{2}} V(\Lambda(z))\right) \vartheta_{1}\right]}{\gamma(z) \vartheta_{2}} p_{1} x z \pi_{0,0}(22)$

$\varphi_{1}(x, z)=\frac{S(x)-S(\Lambda(z))}{x-\Lambda(z)} \frac{z\left[\Lambda(z)+p_{2} V(\Lambda(z))\right] \vartheta_{1}+\left(A\left(\overline{p_{1}}\right)(1-z)+a_{0} z\right) \tau(z)}{\gamma(z) \vartheta_{2}} p_{1} x \pi_{0,0}$

Substituting equations (15) and (16) in equations (12) and (13), we obtain

$\varphi_{2}(x, z)=\frac{V(x)-V(\Lambda(z))}{x-\Lambda(z)} \frac{\vartheta_{1}}{\vartheta_{2}} p_{2} x \pi_{0,0}$

Using the normalization condition

$\pi_{0,0}+\varphi_{0}(1,1)+\varphi_{1}(1,1)+\varphi_{2}(1,1)=1$

we get $\pi_{0,0}=\frac{\left(p_{2}+\bar{p}_{2} A\left(\overline{p_{1}}\right)-\rho\right) \vartheta_{2}}{A\left(\overline{p_{1}}\right)\left(1+p_{2}\right) \vartheta_{1}+p_{2}\left(V_{1}-1\right) \vartheta_{1}}$

\section{Performance Measures}

In the steady state,

1. When theserver is idle, the marginal generating function of the number of customers in the orbit is given by $\pi_{0,0}+\varphi_{0}(1, z)$

$$
=\frac{z[1-A(\bar{p})]\left[\left(p_{2} z-\bar{p}_{2} a_{0}\right)\left(\Lambda(z)-\bar{p}_{2} V(\Lambda(z))\right)+z\left[\Lambda(z)+p_{2} V(\Lambda(z))\right] S(\Lambda(z))-z \Lambda(z)\left(\Lambda(z)-\bar{p}_{2} V(\Lambda(z))\right)\right.}{\gamma(z) \vartheta_{2}}
$$

2. When the server is busy, the marginal generating function of the number of customers in the orbit is given by

$\varphi_{1}(1, z)=\frac{1-S(\Lambda(z))}{1-\Lambda(z)} \frac{z\left[\Lambda(z)+p_{2} V(\Lambda(z))\right] \vartheta_{1}+\left(A\left(\bar{p}_{1}\right)(1-z)+a_{0} z\right) \tau(z)}{\gamma(z) \vartheta_{2}} p_{1} \pi_{0,0}$

3. When the server is on vacation, the marginal generating function of the number of customers in the orbit is given by

$$
\varphi_{2}(1, z)=\frac{1-V(\Lambda(z))}{1-z} \frac{\vartheta_{1}}{\vartheta_{2}} \pi_{0,0}
$$

4. The probability generating function of the number of customers in the orbit(denoted by $\mathrm{N}$ )is given by $\Psi(z)=\pi_{0,0}+\varphi_{0}(1, z)+\varphi_{1}(1, z)+\varphi_{2}(1, z)=$

$$
\frac{z\left[\Lambda(z)+p_{2} V(\Lambda(z))\right] \vartheta_{1}+\left(A\left(\bar{p}_{1}\right)(1-z)+a_{0} z\right) \tau(z)}{\gamma(z) \vartheta_{2}} p_{1} \pi_{0,0}
$$

5. The probability generating function of the number of customers in the system(denoted by $L$ ) is given by $\phi(z)=\pi_{0,0}+\varphi_{0}(1, z)+z \varphi_{1}(1, z)+z \varphi_{2}(1, z)$ $=\frac{z\left[\Lambda(z)+p_{2} V(\Lambda(z))\right] \vartheta_{1}+\left(A\left(\overline{p_{1}}\right)(1-z)+a_{0} z\right) \tau(z)}{\gamma(z)\left[\overline{p_{2}} A\left(\overline{p_{1}}\right)+p_{2}\left(V_{1}-1\right)\right]}\left(p_{2}+\bar{p}_{2} A\left(\overline{p_{1}}\right)-\rho\right) S(\Lambda(z))$

6. The mean number of customers in the orbit is $\mathrm{E}(\mathrm{N})=\Psi^{\prime}(1)=$ $\frac{p_{2}^{2} S_{2}+2 \overline{p_{2}}\left(p_{2}-\rho\right)\left(A\left(\overline{p_{1}}\right)-1\right)}{2\left[p_{2}+\bar{p}_{2} A\left(\overline{p_{1}}\right)-\rho\right]}+\frac{p_{2}^{2} V_{2} \vartheta_{1}+2 \overline{p_{2}}\left(A\left(\overline{p_{1}}\right)-1\right)\left(1-V_{1}\right) p_{2} \vartheta_{1}}{2\left[A\left(\overline{p_{1}}\right)\left(1+p_{2}\right) \vartheta_{1}+p_{2}\left(V_{1}-1\right) \vartheta_{1}\right]}$

7. The mean number of customers in the system is $\mathrm{E}(\mathrm{L})=\phi^{\prime}(z)=\rho+\mathrm{E}(\mathrm{N})$

\section{Stochastic Decomposition}

The stochastic decomposition law has been analysed extensively for the queueing system with server vacations. This property allows to study the system by considering separately the distribution of the system size without vacation and the additional system size due to vacation. In this section, we investigate the stochastic decomposition property for the number of customers in our model.

The total number of customers in the system $\mathrm{L}$ can be decomposed as the sum of two independent random variables $\mathrm{L}=\mathrm{L}_{\mathrm{c}}+\mathrm{L}_{\mathrm{v}} . \mathrm{L}_{\mathrm{c}}$ is the number of customers in the Geo/G/1 queue with state dependent arrival and $\mathrm{L}_{\mathrm{v}}$ is the number of repeated customers given that the server is idle or vacation.

$\phi(z)$ can be expressed as $\phi(z)=\chi_{1}(z) \chi_{2}(z)$, where 


$$
\chi_{1}(z)=\frac{(1-\rho)(1-z) S(\Lambda(z))}{S(\Lambda(z))-z}
$$

is the generating function of standard Geo/G/1/o queue with state dependent arrival and $\chi_{2}(z)$ is given by $\chi_{2}(z)=\frac{S(\Lambda(z))-z}{(1-\rho)(1-z)} \times \frac{z\left[\Lambda(z)+p_{2} V(\Lambda(z))\right] \vartheta_{1}+\left(A\left(\bar{p}_{1}\right)(1-z)+a_{0} z\right) \tau(z)}{\gamma(z) \vartheta_{2}} \times \frac{\left(p_{2}+\bar{p}_{2} A\left(\bar{p}_{1}\right)-\rho\right) \vartheta_{2}}{A\left(\bar{p}_{1}\right)\left(1+p_{2}\right) \vartheta_{1}+p_{2}\left(V_{1}-1\right) \vartheta_{1}}$

It is easy to verify that

$$
\chi_{2}(z)=\frac{\pi_{0,0}+\varphi_{0}(1, z)+\varphi_{2}(1, z)}{\pi_{0,0}+\varphi_{0}(1,1)+\varphi_{2}(1,1)}
$$

which is the generating function of the number of customers in the orbit given that the server is idleor vacation.

\section{Numerical Results}

To study the impact of system parameters on the main performance measures of the system we present some numerical examples in this section. It is assumed that, the retrial times are geometric distributions with parameter $r$ and their generating function is $\mathrm{A}(x)=(1-r) /(1-r x)$. The service time andvacation times are also assumed to follow geometric distributions with parameters $\mu$ and $\mathrm{v}$ respectively.The following notations are used;

Average arrival rate when the system is idle: $p_{1}$

Average arrival rate when the system is busy or on vacation: $p_{2}$

Retrial rate: $r$

Vacation rate: $v$

Table (a) and figure (a) shows the effect of varying values of arrival rate ' $p_{1}$ ' (when the system is idle) on the mean orbit size $\mathrm{E}(\mathrm{N})$. Assume the parameter values as $S_{1}=2 ; S_{2}=6 ; V_{1}=2 ; V_{2}=6 ; \mathrm{r}=0.4 ; \mathrm{v}=0.4$. From the table and figure, it can be observed that the mean orbit size increases when arrival rate $p_{1}$ increases.

Table (b) and figure (b) exhibits the effect of varying values of arrival rate ' $p_{2}$ ' (when the system is busy or on vacation)on the mean orbit size $\mathrm{E}(\mathrm{N})$. Assume the parameter values as $S_{1}=2 ; S_{2}=6 ; V_{1}=2 ; V_{2}=6$; $\mathrm{r}=0.4 ; \mathrm{v}=0.4$. From the table and figure, it can be observed that the mean orbit size increases when arrival rate $p_{2}$ increases.

Table (c) and figure (c) represents the effect of varying values of retrial rate ' $r$ ' on the mean orbit size $\mathrm{E}$ $(\mathrm{N})$. Assume the parameter values as $S_{1}=2 ; S_{2}=6 ; V_{1}=2 ; V_{2}=6 ; \mathrm{v}=0.4 ; p_{2}=0.1 ; \mathrm{v}=0.4$. From the table and figure, it can be observed that the mean orbit size increases when retrial rate increases.

Table :( a) Mean orbit size E (N) vsarrival rate $p_{1}$

\begin{tabular}{|l|l|l|l|}
\hline & & & 0.3 \\
\end{tabular}

Table: (b) Mean orbit size E (N) vs arrival rate $p_{2}$

\begin{tabular}{|l|l|l|l|}
\hline & & & 0.4 \\
& & & \\
\hline 0.10 & 0.1964 & 0.2 & \\
\hline 0.11 & 0.2121 & 0.2627 & 0.3285 \\
\hline 0.12 & 0.2299 & 0.2794 & 0.3463 \\
\hline 0.13 & 0.2497 & 0.2984 & 0.3665 \\
\hline 0.14 & 0.2719 & 0.3197 & 0.3894 \\
\hline
\end{tabular}


Analysis of A Discrete- Time Single Server Retrial Queue with General Retrial Times, Multiple ..

\begin{tabular}{|l|l|l|l|}
\hline 0.15 & 0.2965 & 0.3702 & 0.4439 \\
\hline 0.16 & 0.3236 & 0.3998 & 0.4760 \\
\hline 0.17 & 0.3536 & 0.4326 & 0.5116 \\
\hline 0.18 & 0.3867 & 0.4688 & 0.5513 \\
\hline 0.19 & 0.4231 & 0.5089 & 0.5953 \\
\hline 0.20 & 0.4632 & 0.5532 & 0.6442 \\
\hline
\end{tabular}

Table : ( c) Mean orbit size $\mathrm{E}(\mathrm{N})$ vs retrial rate $\mathrm{r}$

\begin{tabular}{|l|l|l|l|}
\hline & & 0.5 & 0.6 \\
\end{tabular}

Figure : ( a )Mean orbit size E (N) vs arrival rate $p_{1}$

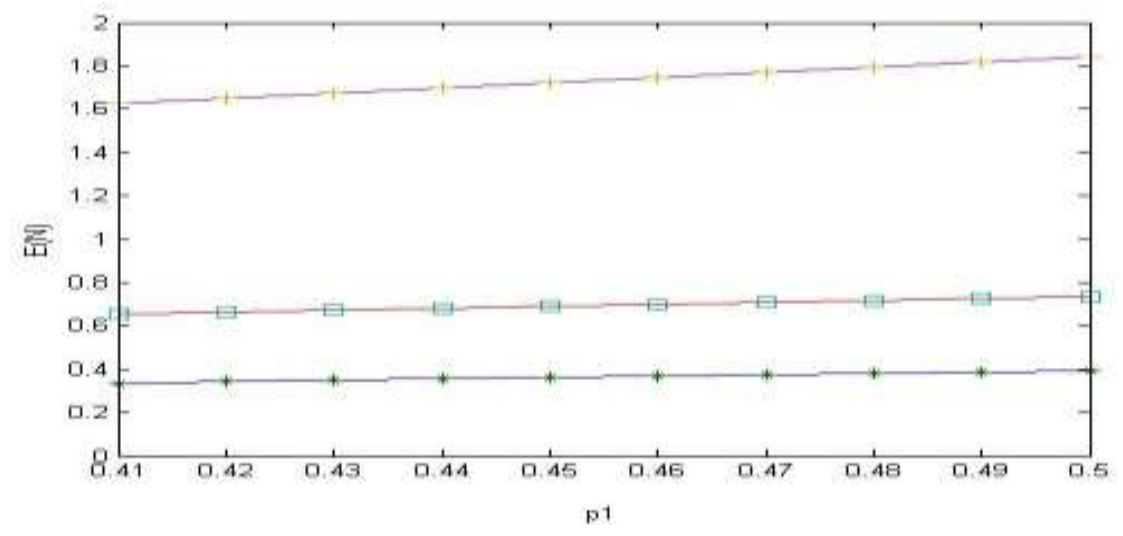

Figure: (b)Mean orbit size E (N) vs arrival rate $p_{2}$

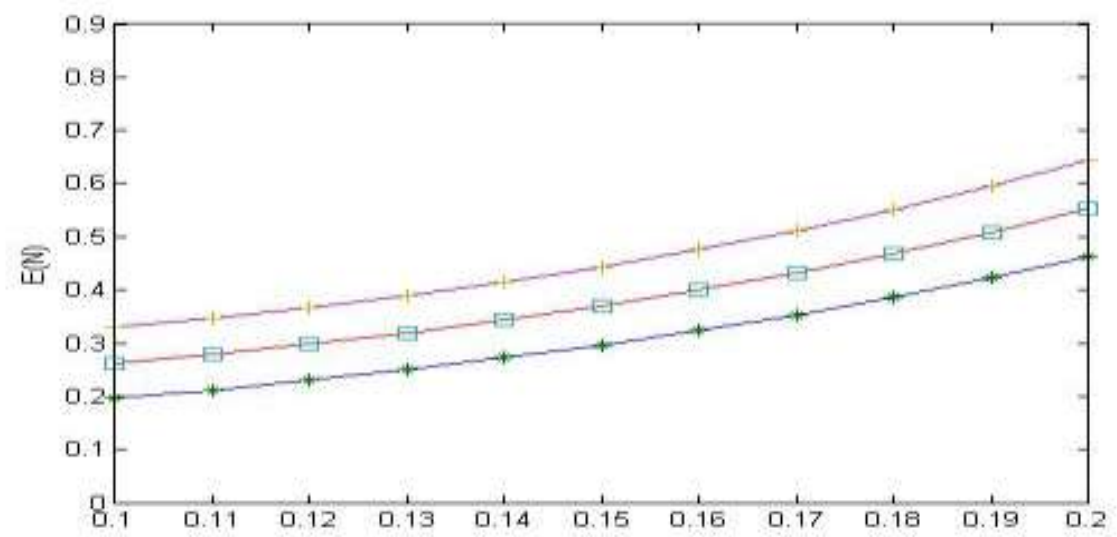

p2 
Figure :(c) Mean orbit size $\mathrm{E}(\mathrm{N})$ vs retrial rate $\mathrm{r}$

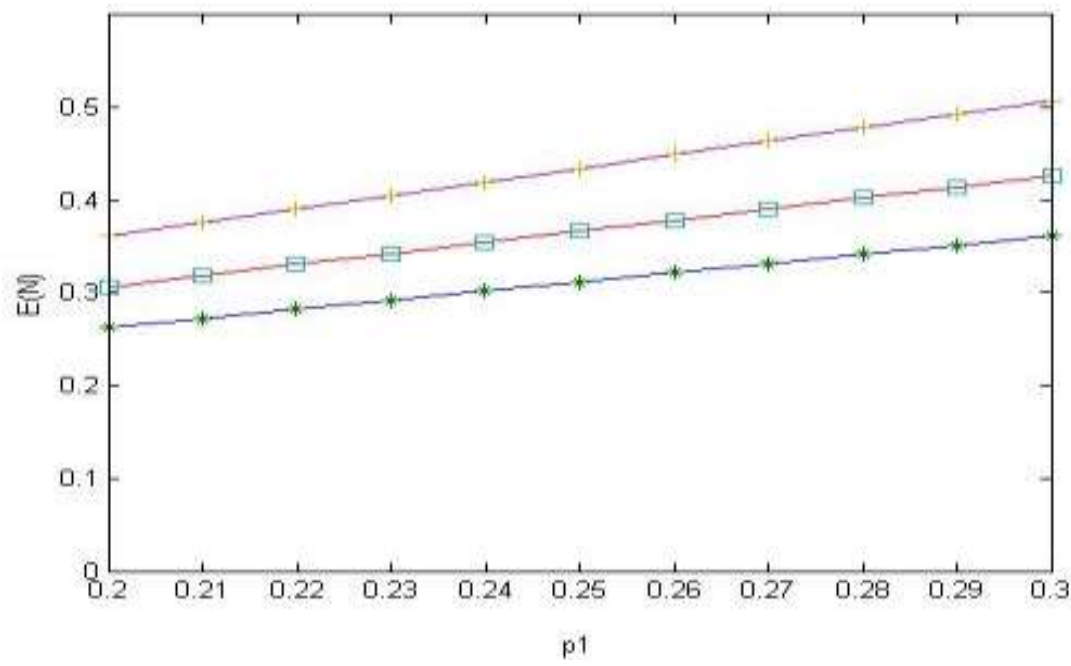

VI. Conclusion

In this paper a discrete time $\mathrm{Geo} / \mathrm{G} / 1$ retrial queue with general retrial times, multiple vacations and state dependent arrivals is analysed under stability condition. Several performance measures are computed in steady state. Some numerical illustrations are also presented to study the effect of parameters on several performance characteristics.

\section{References}

[1]. J.R. Artalejo, Accessible bibliography on retrial queues, Math. Computer Model. 30, 1999, 1-6.

[2]. J.R. Artalejo, A classified bibliography of research on retrial queues: Progress in 1990-1999, Top7, 1999, 187-211.

[3]. J.R. Artalejo, Accessible bibliography on retrial queues: progress in 2000-2009, Math. Computer. Model, 51, 2010, 1071-1081.

[4]. J.R. Artalejo, A. Gomez-Corral, Retrial Queueing Systems: A Computational Approach, (Springer,Berlin, 2008).

[5]. J.R. Artalejo, Analysis of an M/G/1 queue with constant repeated attempts and server vacations, Computers and Operations Research, 1997, 24, 493-504.

[6]. J.R. Artalejo and G. I. Falin, Stochastic decomposition for retrial queues, Top, 1994, 2, 329-342.

[7]. P.Atencia, and Moreno, A discrete-time Geo/G/1 retrial queue with general retrial times, QueueingSystem, 48,2004,5-21.

[8]. G.I. Falin, A survey of retrial queues, Queueing System. 7, 1990, 127-167.

[9]. G.I. Falin, J.G.C. Templeton, Retrial Queues, (Chapman \& Hall, London, 1997)

[10]. J. J. Hunter, Mathematical Techniques of Applied Probability, Discrete Time Models: Techniques and Applications, (Volume 2, Academic Press, 1983).

[11]. V.Jailaxmi and R.Arumuganathan, Analysis of a single server retrial queue with multiplevacations and state dependent arrival,RAIRO-Operations Research, 49, 2015, 619-634.

[12]. J. C.Ke and Y. K.Chu, A modified vacation model M丷/G/1 system, Applied Stochastic Models inBusiness and Industry, 22,2006,116.

[13]. J C Ke and F M Chang, Modified vacation policy for M/G/1 retrial queue with balking andfeedback, Computers and Industrial Engineering, 57, 2009, 433-443.

[14]. H. Li, T. Yang, Geo/G/1 discrete time retrial queue with Bernoulli schedule, European Journal ofOperations Research, 111,1998,629-649.

[15]. H. Li and T. Yang, A single-server retrial queue with server vacations and a finite number of input sources, European Journal of Operational Research, 85, 1995, 149-160.

[16]. Takagi, H.,Queueing Analysis: A Foundation of Performance Evaluation,(Discrete-time Systems, Vol.3, 1993,NorthHolland,Amsterdam).

[17]. N. Tian and Z G Zhang, Vacation Queueing Models: (Theory and Applications. Springer, 2006).

[18]. T. Yang, J.G.C. Templeton, A survey on retrial queues, Queueing System. 2, 1987, 201-233.

[19]. Z. G. Zhang and N. Tian, Discrete time Geo/G/1 queue with multiple adaptive vacations, Queueing Systems, vol. 38, no. 4, 419429, 2001 